\title{
DISSIPATIVE PERIODIC PROCESSES
}

\author{
BY J. E. BILLOTTI ${ }^{1}$ AND J. P. LASALLE ${ }^{2}$
}

Communicated by Felix Browder, June 17, 1971

1. Introduction. There has been recently the development of a general theory of dynamical systems going beyond ordinary differential equations which includes functional differential equations, partial differential equations, systems arising in the theory of elasticity, etc. A large number of examples of such dynamical systems and more complete references can be found in the paper [1] by Hale. For extensions to periodic systems and certain nonautonomous systems see [2] and [3]. Applications can be found in [4]-[7].

In this same spirit we develop here a general theory of dissipative periodic systems that applies to systems which "smooth" initial data (retarded functional differential equations, for example). This extends the work of Billotti in [8]. Nonlinear ordinary differentials which are periodic and dissipative were studied by Levinson in [9] in 1944, and more general results can be found in [10] and [11]. For ordinary differential equations one studies the iterates of a map $T$ of a state space into itself where the map $T$ is topological and the space is locally compact ( $n$-dimensional Euclidean space). However, for the applications we have in mind the solutions will be unique only in the forward direction of time and the state spaces are not locally compact. Because of this the generalization of the results for ordinary differential equations is by no means trivial.

The basic theory of disspative periodic processes on Banach spaces is developed in $\$ \S 2$ and 3 . How this applies to retarded functional differential equations of retarded type is discussed briefly in $\$ 4$.

2. Dissipative mappings. Let $R$ denote the real numbers, $R^{+}$the nonnegative reals, and let $X$ be a Banach space with norm $\|\cdot\|$.

AMS 1970 subject classifications. Primary 34C35, 34J05; Secondary 34K15, 34K25.

Key words and phrases. Dissipative periodic processes, periodic dynamical systems, discrete dynamical systems, functional differential equations, periodic solutions, fixed point property, stability, invariance, nonlinear oscillations, steady state solution.

1 This research was supported by the National Aeronautics and Space Administration under Grant No. NGL 40-002-015.

2 This research was supported in part by the National Aeronautics and Space Administration under Grant No. NGL 40-002-015, in part by the Air Force Office of Scientific Research under Grant No. AF-AFOSR 67-0693A, and by the United States Army-Durham under Grant No. DA-31-124-ARO-D-270. 
Consider a mapping $u: R \times X \times R^{+} \rightarrow X$ and define $(t, \tau): X \rightarrow X$ for each $t \in R$ and each $\tau \in R^{+}$by $(t, \tau) x=u(t, x, \tau)$. Interpret $(t, \tau) x$ as the state of the system at time $t+\tau$ if initially the state of the system at time $t$ was $x$. A process on a Banach space $X$ is a mapping $u: R \times X$ $\times R^{+} \rightarrow X$ with the following properties: (2.1) $u$ is continuous, (2.2) $(t, 0) x=x,(2.3)(t+\sigma, \tau)(t, \sigma)=(t, \sigma+\tau)$. Thus a process here is essentially what was called in [2] a "generalized nonautonomous dynamical system" and differs by the continuity on $u$ from what was called a process in [3].

A process is said to be periodic of period $\omega>0$ if $(t+\omega, \tau)=(t, \tau)$ for all $t \in R$ and all $\tau \in R^{+}$. For any fixed $t \in R$ there is then associated with a periodic process a continuous mapping $T: X \rightarrow X$ defined by $T(x)=(t, \omega) x$. With $T^{n}$ the $n$th iterate of $T$ it follows from (2.3) that $T^{n}(x)=(t, n \omega) x$ and the sequence $T^{n}(x), n=0,1,2 \ldots$, is called the (positive) motion or orbit through $x$. Since for a periodic process $(t, \tau+k \omega)=(t, \tau)(t, k \omega)$, we see that the fixed points of $T^{k}$ correspond to periodic motions of the periodic process.

Thus motivated we will now spend the rest of this section studying the discrete dynamical system defined by an arbitrary continuous mapping $T: X \rightarrow X$ where $X$ is a Banach space. A point $y$ is said to be a limit point of the motion $T^{n}(x)$ if there exists a subsequence $n_{k}$ of integers such that $n_{k} \rightarrow \infty$ and $T^{n_{k}}(x) \rightarrow y$ as $k \rightarrow \infty$. The limit set $L(x)$ is the set of all limit points of $T^{n}(x)$. Note that $L(x)=\bigcap_{j=0}^{\infty} \mathrm{Cl} \cup_{n=j}^{\infty} T^{n}(x)$, where $\mathrm{Cl}$ is closure.

A set $M \subset X$ is said to be positively invariant if $T(M) \subset M$ and negatively invariant if $M \subset T(M)$. It is said to be invariant if $T(M)=M$; i.e., if it is both positively and negatively invariant. Negative invariance implies the existence of an extension over all integers of each positive motion through a point of $M$ and the negative extension is contained in $M$.

Lemma 2.1. If the motion $T^{n}(x), n=0,1,2, \cdots$, is precompact, then the limit set $L(x)$ is nonempty, compact and invariant.

For most applications other than ordinary differential equations the state space $X$ is not locally compact and there is the practical difficulty of determining compactness. For many processes $T$ smooths the initial data and with suitable topologies for the state spaces boundedness of the motion implies that the motion is precompact (see, for example, [1]). With applications in mind we develop a theory of dissipative processes based on boundedness and require a smoothing property stronger than that mentioned above.

Definition 2.1. $T$ is said to smooth if there is a nonnegative integer 
$n_{0}$ such that for each bounded set $B$ in $X$ there is a compact set $B^{*}$ in $X$ such that $T^{n}(x) \in B$, for $n=0,1, \cdots, N\left(N \geqq n_{0}\right)$, implies $T^{n}(x) \in B^{*}$ for $n=n_{0}, n_{0}+1, \cdots, N$.

For ordinary differential equations every continuous $T$ smooths with $n_{0}=0$ ( $X$ is locally compact) and for retarded functional differential equations $T$ smooths with $n_{0} \omega \geqq r$ ( $\omega$ the period and $r$ the retardation).

Definition 2.2. $T$ is dissipative if (1) it smooths and (2) there is a bounded set $B$ in $X$ with the property that given $x \in X$ there is a positive integer $N(x)$ such that $T^{n}(x) \in B$ for $N(x) \leqq n \leqq N(x)+n_{0}$.

This next result generalizes Theorems 2.1 and 2.2 of [11] and here the proofs are both simpler and more elegant. If the space is locally compact (ordinary differential equations), then every continuous $T$ smooths and $T$ is dissipative if there is a bounded set $B$ such that for each $x \in X$ there is an $N(x)$ such that $T^{N(x)}(x) \in B$. If the space is not locally compact, the assumption that $T$ smooths is needed and for each $x \in X$ the motion $T^{n}(x)$ must remain in $B$ long enough to smooth.

THeOREM 2.1. If $T$ is dissipative, then there is a compact set $K$ in $X$ with the property that given a compact set $H$ in $X$ there is a positive integer $N(H)$ and an open neighborhood $O_{H}$ of $H$ such that $T^{n}\left(O_{H}\right) \subset K$ for all $n \geqq N(H)$.

The principle result is an easy consequence of the Schauder fixed point theorem.

Corollary 2.1. If $T$ is dissipative, $T^{j}$ has a fixed point for each integer $j$ greater than some integer $k$.

If $T$ maps bounded sets into bounded sets, then using Browder's extension [12] of the Schauder fixed point theorem one obtains:

Corollary 2.2. If $T$ is dissipative and maps bounded sets into bounded sets, then $T^{j}$ has a fixed point for each integer $j \geqq n_{0}$.

For ordinary differential equations and for retarded functional differential equations with $\omega \geqq r$ ( $\omega$ is the period and $r$ is the retardation), $n_{0}=1$ and the conclusion is that $T$ itself has a fixed point. This was shown by Yoshizawa (see [13] or [14]) for periodic retarded functional differential equations if the solutions are uniformly bounded and uniformly ultimately bounded. The above corollary includes Yoshizawa's result. Under even stronger conditions this problem has been studied by Jones in [15] and [16]. One suspects that $T$ being dissipative would imply that $T$ has a fixed point but this is at the moment merely a conjecture. For an example of a retarded 
functional differential equation where bounded sets are not mapped into bounded sets by the flow defined by solutions see [17].

There is a very special class of dissipative systems where $T$ has a unique fixed point. In the theory of oscillations this unique fixed point corresponds to the "steady state" oscillation. For a topological map $T$ and hence for periodic ordinary differential equations a result of this type was given in [18, Corollary 2]. (For ordinary differential equations see also [11] and for retarded differential equations see [19].)

Definition 2.3. $T$ is said to be extremely stable if (1) there is a bounded motion $x, T(x), \cdots, T^{n}(x), \cdots$ and $(2)\left\|T^{n}(x)-T^{n}(y)\right\| \rightarrow 0$ as $n \rightarrow \infty$ for each $x, y \in X$.

CoROLlaRy 2.3. If $T$ is smooth and extremely stable, then $T$ has a unique fixed point which is a global attractor.

3. The limit set $I$. We wish now to point out that if $T$ is dissipative then there is a compact invariant set $I$ that is globally asymptotically stable. Just as in [9] for second order ordinary differential equations $I$ will be the maximum compact set invariant under $T$.

Let $K$ be the compact set of Theorem 2.1. Define $I=\bigcap_{n=0}^{\infty} T^{n}(K)$. Of course, $K$ is not unique, but it is not difficult to see that $I$ does not depend on $K$.

It is interesting to relate $I$ to the motion $K, T(K), \cdots$, $T^{n}(K), \cdots$ Given a set $H$ in $X$ we define $L(H)$, called the limit set of the motion through $H$, by $L(H)=\bigcap_{j=0}^{\infty} \mathrm{Cl} \cup_{n=j}^{\infty} T^{n}(H)$, where $\mathrm{Cl}$ denotes closure. Then $y \in L(H)$ means there exist sequences $n_{i}$ and $y_{i} \in H$ such that $n_{i} \rightarrow \infty$ and $T^{n_{i}}\left(y_{i}\right) \rightarrow y$ as $i \rightarrow \infty$. Thus when $H$ is a single point $x$ this is the usual limit set $L(x)$. Now just as for Lemma 2.1 it follows that

LEMмA 3.1. If for some $j$ sufficiently large $\bigcup_{n=j}^{\infty} T^{n}(H)$ is precompact, then the limit set $L(H)$ is nonempty, compact, and invariant.

Theorem 3.1. Assume that $T$ is dissipative. Then $I=L(K)$ and hence $I$ is nonempty, compact, invariant and is the maximum compact invariant set in $X$.

We recall that a set $M$ is a global attractor if $T^{n}(x) \rightarrow M$ as $n \rightarrow \infty$ for each $x \in X$. Since each motion $T^{n}(x)$ is precompact (Theorem 2.1 ) and its limit set $L(x)$ is nonempty, compact and invariant (Lemma 2.1), it follows from the above theorem that $L(x)$ is in $I$ for each $x \in X$. Hence $I$ is a global attractor. For $\delta>0$ let $M^{\delta}$ denote the $\delta$-neighborhood of $M\left(M^{\delta}=\{y ;\|y-x\|<\delta\right.$ for some $\left.x \in M\}\right)$. A 
set $M$ is said to be stable if given $\epsilon>0$ there is a $\delta>0$ such that $x \in M^{\delta}$ implies that $T^{n}(x) \in M^{\epsilon}$ for all $n \geqq 0$. If the set $M$ is both stable and a global attractor it is said to be globally asymptotically stable. By an argument similar to that used by LaSalle to prove Theorem 3 in $[20]$ it follows that

Theorem 3.2. If $T$ is dissipative, then the set $I$ is globally asymptotically stable.

4. Retarded functional differential equations. We examine briefly how $\$ \S 2$ and 3 can be applied to retarded functional differential equations. Let $R^{n}$ be a real $n$-dimensional vector space with norm $|\cdot|$. Given $r>0, C=C\left([-r, 0], R^{n}\right)$ will denote the space of continuous functions $\phi$ mapping $[-r, 0]$ into $R^{n}$ with $\|\phi\|$ $=\sup \{\phi(\theta) ;-r \leqq \theta \leqq 0\}$. Let $f$ be a continuous function taking $R \times C$ into $R^{n}$. A retarded functional differential equation is a system of the form

(4.1) $\dot{x}(t)=f\left(t, x_{t}\right)$, where $\dot{x}$ is the derivative of $x$ and $x_{t} \in C$ is defined by $x_{t}(\theta)=x(t+\theta),-r \leqq \theta \leqq 0$. A function $x$ mapping $\left[t_{0}-r, t_{0}+a\right)$ into $R^{n}$ is said to be a solution of (4.1) on $\left[t_{0}, t_{0}+a\right)$ with initial value $\phi \in C$ at $t_{0}$ if $x$ has a continuous derivative on $\left[t_{0}, t_{0}+a\right)$ satisfying (4.1) and $x_{t 0}=\phi$.

A brief survey of the history of functional differential equations is given in [21]. For general theorems on existence, uniqueness, continuation and continuity see $[1],[4],[14],[22]$, or [23]. These theorems are quite similar to those for ordinary differential equations, and we make the general assumption that $f$ satisfies, in addition to the continuity condition above, conditions sufficient to insure uniqueness of solutions to the right. We shall also assume that the solution $x\left(t, t_{0}, \phi\right)$ of $(4.1)$ satisfying $x_{t_{0}}\left(t_{0}, \phi\right)=\phi$ is defined for all $t \geqq t_{0}$. This will be implied by dissipativeness. Then $u\left(t_{0}, \phi, \tau\right)=x_{t_{0}+\tau}\left(t_{0}, \phi\right)$ is, as described in $\$ 2$, a process on the Banach space $C$. We shall assume also (1) $f(t, \phi)$ is periodic in $t$ with period $\omega>0$ and (2) $f$ maps bounded sets of $R \times C$ into bounded sets of $R^{n}$. If $x(t)$ is any solution of (4.1), we see that $|x(t)|<b$ for $t \in\left[t_{0}, t_{0}+T\right)$ implies $\left\|\dot{x}_{t}\right\|<d$ for $t \in\left[t_{0}+r, t_{0}+T\right)$. Thus corresponding to each bounded set $B$ in $C$ there is a compact set $B^{*}$ in $C$ such that $x_{t} \in B$ for $t \in\left[t_{0}, t_{0}+T\right)$ implies $x_{t} \in B^{*}$ for $t \in\left[t_{0}+r, t_{0}+T\right)$. This smoothing of the initial data was exploited by Hale [1] although he did not use and did not need a smoothing property as strong as this one. Defining $T(\phi)=x_{t_{0}+\omega}\left(t_{0}, \phi\right)$ for any fixed $t_{0}$, we see that $T$ smooths in the sense of Definition 2.1 with $n_{0}$ the least integer such that $n_{0} \omega \geqq r$. We see also that $T$ will be 
dissipative if there is a number $b$ such that given $\phi \in C$ there is a $t_{1}=t_{1}\left(\phi, t_{0}\right)$ with the property that $\left|x\left(t, t_{0}, \phi\right)\right|<b$ for all $t_{1} \leqq t \leqq t_{1}+n_{0} \omega$.

\section{REFERENCES}

1. J. K. Hale, Dynamical systems and stability, J. Math. Anal. Appl. 26 (1969), 39-59. MR $39 \# 5896$.

2. M. Slemrod, Asymptotic behavior of periodic dynamical systems on Banach spaces, Ann. Mat. Pura Appl. (to appear).

3. C. M. Dafermos, An invariance principle for compact processes, J. Differential Equations 9 (1971), 239-252.

4. M. A. Cruz and J. K. Hale, Stability of functional differential equations of neutral type, J. Differential Equations 7 (1970), 334-355. MR 41 \#2166.

5. M. Slemrod and E. F. Infante, An invariance principle for dynamical systems on Banach spaces: Application to the general problem of thermoelastic stability, Proc. IUTAM Sympos. on Instability, Springer-Verlag, Berlin and New York (to appear).

6. C. M. Dafermos, Asymptotic stability in viscoelasticity, Arch. Rational Mech. Anal. (to appear).

7. M. Slemrod, Nonexistence of oscillations in a nonlinear distributed network, J. Math. Anal. Appl. (to appear).

8. J. E. Billotti, Dissipative functional differential equations, Ph.D. Dissertation, Brown University, Providence, R. I., 1969.

9. Norman Levinson, Transformation theory of non-linear differential equations of the second order, Ann. of Math. (2) 45 (1944), 723-737. MR 6, 173.

10. R. Ressig, G. Sansone and R. Conti, Nichtlineare Differential gleichungen höhrer Ordnung, Edizioni Cremonese, Roma, 1969.

11. V. A. Pliss, Nonlocal problems of the theory of oscillations, "Nauka", Moscow, 1964; English transl., Academic Press, New York, 1966. MR 30 \#2188; 33 \#4391.

12. F. E. Browder, On a generalization of the Schauder fixed point theorem, Duke Math. J. 26 (1959), 291-303. MR 21 \#4368.

13. T. Yoshizawa, Ultimate boundedness of solutions and periodic solution of functional-differential equations, Colloq. Internat. Vibrations Forcées dans les Systémes Non-Linéaires, Marseilles, 1964, pp. 167-179.

14. - - Stability theory by Liapunov's second method, Publ. Math. Soc. Japan, no. 9, Math. Soc. Japan, Tokyo, 1966. MR 34 \#7896.

15. G. S. Jones, Asymptotic fixed point theorems and periodic systems of functional differential equations, Contributions to Differential Equations 2 (1963), 385-405. MR $28 \# 1361$.

16. - Periodic motions in Banach space and applications to functional differential equations with retarded arguments, Contributions to Differential Equations 3 (1963), 75-106.

17. J. K. Hale, Functional differential equations, Proc. Conference Anal. Theory of Differential Equations (Kalamazoo, Michigan, 1970), Springer-Verlag, New York (to appear).

18. J. P. LaSalle, A study of synchronous asymptotic stability, Ann. of Math (2) 65 (1957), 571-581. MR 19, 35.

19. T. Yoshizawa, Extreme stability and almost periodic solutions of functional differential equations, Arch. Rational Mech. Anal. 17 (1964), 148-170. MR 29 \#3734. 
20. J. P. LaSalle, Stability theory for ordinary differential equations, J. Differential Equations 4 (1968), 57-65. MR 36 \#5454.

21. J. K. Hale, Geometric theory of functional-differential equations, Proc. Internat. Sympos. Differential Equations and Dynamical Systems (Mayaguez, P. R., 1965), Academic Press, New York, 1967, pp. 247-266. MR 36 \#5465.

22. V. Lakshmikantham and S. Leela, Differential and integral inequalities. Vol. II, Academic Press, New York, 1969.

23. J. K. Hale, Lectures on functional differential equations, University of California, Los Angeles, Calif., 1968/69.

LeMoyne College, Syracuse, New York 13214

Brown University, Providence, Rhode Island 02912 